\title{
Evaluation of the Visual Navigation Chart Based on Visual Perception
}

\author{
Hong-yong Wang ${ }^{1, a}$, Peng $\mathrm{Li}^{2, \mathrm{~b}}$ and Yi-fei Zhao ${ }^{3, \mathrm{c}}$ \\ ${ }^{1}$ Air Traffic Management Research Base, Civil Aviation University of China, Tianjin, China \\ ${ }^{2}$ Air Traffic Management Research Base, Civil Aviation University of China, Tianjin, China \\ ${ }^{3}$ Air Traffic Management Research Base, Civil Aviation University of China, Tianjin, China \\ achinaATM@yahoo.cn, ${ }^{b}$ pli yjs10@cauc.edu.cn, ${ }^{c} y i f e i 6666 @$ sina.com
}

Key words: VFR chart; Symbol; Visual perception; Fuzzy evaluation

\begin{abstract}
A complete set of symbols is not formed for VFR chart in our country for the slow development of general aviation. On the basis of the VFR chart collected from lots of designers, evaluation index of VFR chart was defined with the Cognitive semiotics and the demand of chart for VFR. All the sample data of the evaluation index was get through the visual perception test. Finally, the value of comprehensive evaluation was given by Fuzzy evaluation .The designing principal of VFR chart symbols was summarized. It provided the theory basis for the development of VFR chart.
\end{abstract}

\section{Introduction}

Visual flight rules chart is a thematic map under the rules of visual flight rules used by pilots. It is mainly used for agriculture and industry, emergency rescue and disaster relief, remote sensing, surveying and mapping, tourism, and other aspects of the civil aviation activities. It is the important condition for small aircraft to securely fly at low altitude. VFR chart has been widely used in general aviation developed countries of Europe and America, In China general aviation overall development lags behind, the VFR chart product is basic blank. Along with the rapid development of general aviation in China. The development of VFR chart that is suitable for civil aviation characteristics and requirements of our country is a important mission in civil aviation navigation chart production department.

At present, the evaluation of VFR chart not only lacks specific assessment and system analysis that closely related to the visual quality of users, but also does not put forward relevant evaluation index. In foreign country, Michelle Yeh, and Divya C. Chandra have made a summary of the current VFR chart symbol, and then only made a evaluation of symbol shapes[1,2];In our country, MENG Ai-min introduced the highly developed VFR chart of USA[3], ZHENG Xin-ming has made a simple comparison of VFR chart elements between European and USA, and described some theoretical issues that need to be paid attention to in the production of VFR chart[4,5]. Therefore, This article will focus on the users and design visual perception test to analyze the requirements of information transmission terminal, information transmission terminal, especially analyze visual quality, and discuss the design of the VFR map symbol design in the visual experience of VFR chart.

\section{Semiotics theory}

The graphic semiotics point of Semiotic study is mainly based on the Belting visual variable theory .Visual variable theory provide a theoretical basis for the symbolic content of the VFR charts, and also played a role in promoting systematization and standardization of the design in the aeronautical chart symbols.

Visual variables of symbol. Visual variables, also known as graphic variable, refers to the change of factors, such as graphics and color, that can cause visual differences between the graphical symbols. France Map scientist Belting extracted the basic elements that constituted the graphic symbol, called "visual variables", including shape, size, color, brightness, texture and direction. They appear in the form of point, line and surface, Belting's definition of visual variables is for generalized graphic symbols, not fully applicable for VFR chart. Some visual variable is rarely used in the symbolic design 
of VFR charts, like most point symbols and line symbols, because the area of the symbol is lesser, the change of texture is very hard to distinguish out, the direction variables in the map design are rarely used. Gittin S professor also puts forward six factors (can be associated, can be identified, univocal, simple design, noticeable, symbolic) used to evaluate symbol[6], This paper consider the following several visual variables: shape, size, color, brightness.

Relevant factors of symbol design. Aeronautical chart symbol is a special graphic symbols, which indicate the nature and mutual relationship of various objects and phenomena in the way of convenient for space positioning. Aeronautical charts symbols have many kinds and its size, shape, orientation, etc. have a direct impact on the appearance of the whole aeronautical chart, So the design of aeronautical charts symbols must take into account the theme and content of the chart, and the reader's psychological and social environmental factors. Its associated factors are shown in Fig 1.

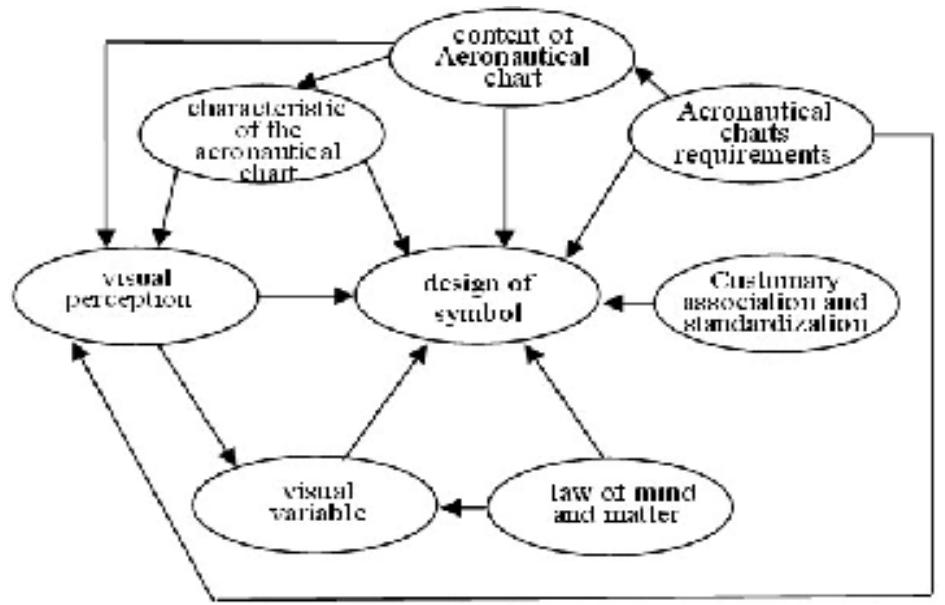

Fig.1 Factors of symbol design

Evaluation index of symbol. Based on the above semiotics, the evaluation index of the whole chart and single symbol is proposed, as shown in Fig 2 and Fig 3.

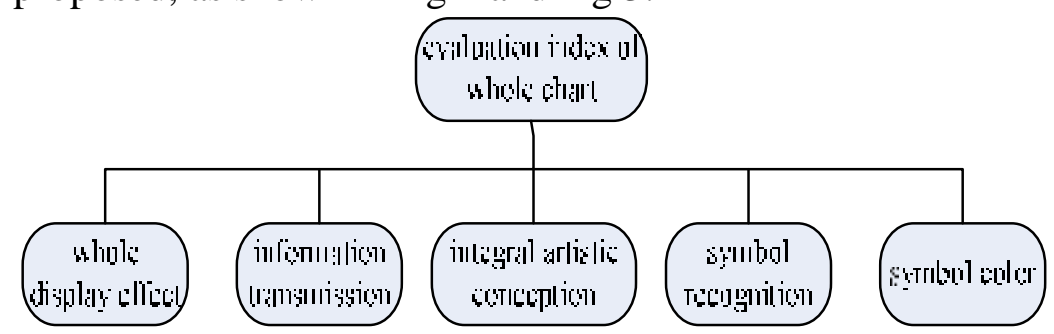

Fig.2 Evaluation index of whole chart

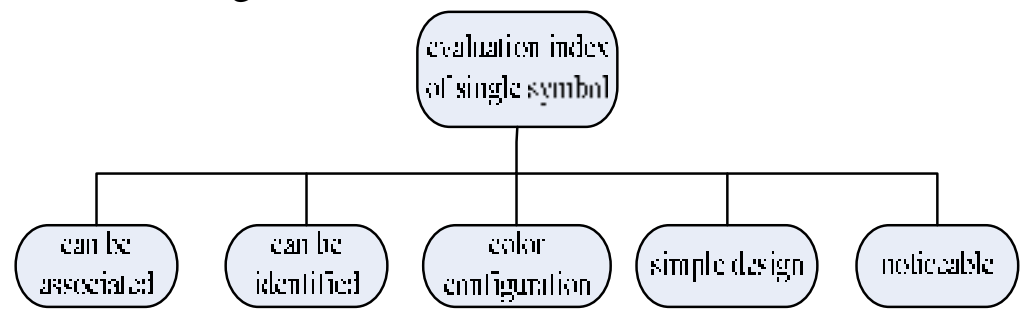

Fig.3 Evaluation index of single symbol

\section{Visual perception test}

Based on the above evaluation index of the whole chart and a single symbol, visual perception test is designed[7]. Then we can get the sample data of all the evaluation index, which are used for the analysis later. It provides a theoretical basis for the development of Chinese VFR charts

The article contains the visual perception test of the whole chart and single symbol, pilots are organized to carefully observe and research the collected VFR charts and the symbols intercepted from the above charts, then gives the assessed value of each index. The test select 4 grade evaluation value they are poor, fair, good and excellent, in turns the numbers 1,2,3and 4 stand for them. That is, the 
greater of the value the better of the evaluation. After the arrangement of experiment data, the results are given by method of fuzzy evaluation.

The experiment selects 30 students as subjects, who have attended flight training from foreign aviation school. Aeronautical charts and symbols of the experiment was provided from the following units, FAA, ICAO, SMATSAICAO(SMATSA), JEPPESEN,AIR Navigation (AIR)and CORPORATE Smalltalk(CORPORATE).The symbols intercepted from the above charts are airport symbol, obstacle symbol, NDB symbol and VORDME symbol. Fig 4 and Fig 5 are the part of the experiment interface.

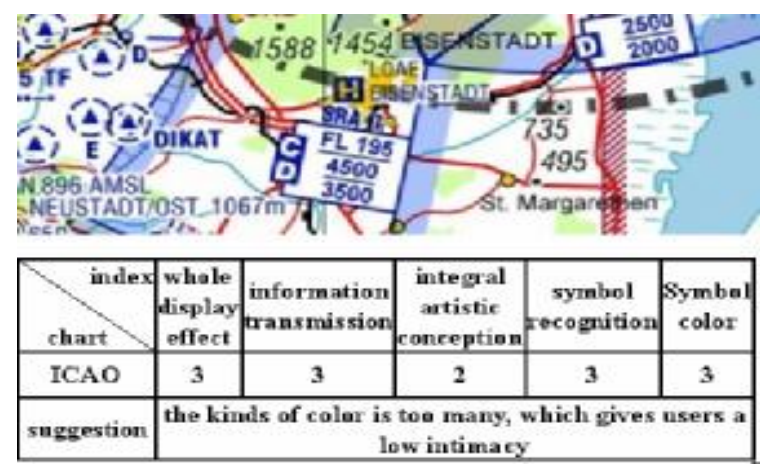

Fig.4 Experimental interface of whole chart
Airport symbol

\begin{tabular}{|c|c|c|c|c|c|}
\hline iudex & $\begin{array}{c}\text { can be } \\
\text { associated }\end{array}$ & $\begin{array}{c}\text { can be } \\
\text { identified }\end{array}$ & $\begin{array}{c}\text { color } \\
\text { configuration }\end{array}$ & $\begin{array}{c}\text { simple } \\
\text { design }\end{array}$ & $\begin{array}{c}\text { Notice } \\
\text { able }\end{array}$ \\
\hline & 3 & 2 & 3 & 2 & 3 \\
\hline & 4 & 3 & 3 & 3 & 3 \\
\hline
\end{tabular}

Fig.5 Experimental interface of single symbol

\section{Fuzzy evaluation}

This experiment applies the method of fuzzy mathematics to establish fuzzy comprehensive evaluation model for most of the evaluation of subjective index and quantify the fuzzy factors. Determine the weight of the evaluation index according to one for comparison method. The vector product was used to work out the numerical generation results of the comprehensive evaluation. This gives a direct comparison of comprehensive evaluation results of the evaluation object, which provides a viable scientific method for the evaluation of VFR chart symbol.

Fuzzy Evaluation Model. Based on the above analysis, the domain of evaluation factors of whole chart is $U=\left\{u_{1}, u_{2}, u_{3}, u_{4}, u_{5}\right\}$, namely, $u_{1}$ represents the index of whole display effect, $u_{2}$ represents the index of information transmission, $u_{3}$ represents the index of integral artistic conception, $u_{4}$ represents the index of symbol recognition, $u_{5}$ represents the index of symbol color. While the domain of evaluation factors of single symbol is $U=\left\{u_{1}, u_{2}, u_{3}, u_{4}, u_{5}\right\}$,namely, $u_{1}$ represents the index of can be associated, $u_{2}$ represents the index of can be identified, $u_{3}$ represents the index of color configuration, $u_{4}$ represents the index of simple design, $u_{5}$ represents the index of noticeable.

The evaluation level of the VFR chart can be divided into 4 level, namely excellent, good, fair, and poor. The domain constitute by evaluation level is $V=\left\{v_{1}, v_{2}, v_{3}, v_{4}\right\}$, namely, $v_{1}$ represents excellent, $v_{2}$ represents good, $v_{3}$ represents fair, $v_{4}$ represents poor. The users give the level of all evaluation factors by experiment.

The evaluation of whole display effect $\left(R_{1}\right)$, information transmission $\left(R_{2}\right)$, integral artistic conception $\left(R_{3}\right)$, symbol recognition $\left(R_{4}\right)$ and Symbol color $\left(R_{5}\right)$ are shown fromFq. 1 to Eq.5.

$R_{1}=\left\{v_{11}, v_{12}, v_{13}, v_{14}\right\}$
$R_{2}=\left\{v_{21}, v_{22}, v_{23}, v_{24}\right\}$
$R_{3}=\left\{v_{31}, v_{32}, v_{33}, v_{34}\right\}$
$R_{4}=\left\{v_{41}, v_{42}, v_{43}, v_{44}\right\}$
$R_{5}=\left\{v_{51}, v_{52}, v_{53}, v_{54}\right\}$

The matrix they constitute is shown in Eq.6. 


$$
R=\left[\begin{array}{llll}
v_{11} & v_{12} & v_{13} & v_{14} \\
v_{21} & v_{22} & v_{23} & v_{24} \\
v_{31} & v_{32} & v_{33} & v_{34} \\
v_{41} & v_{42} & v_{43} & v_{44} \\
v_{51} & v_{52} & v_{53} & v_{54}
\end{array}\right]
$$

Determine the weights of evaluation factors, and the evaluation index of the two above experiments is not treated equally. In this article, the method to determine by two experimental .Index weights and weight value is as follow:

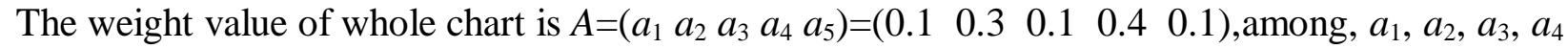
and $a_{5}$ in turn represent the weight value of display effect, information transmission, integral artistic conception, symbol recognition and Symbol color.

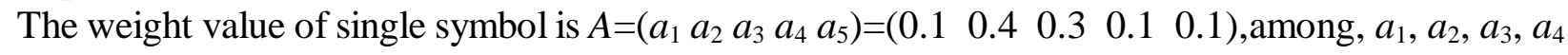
and $a_{5}$ in turn represent the weight value of can be associated, can be identified, color configuration, simple design and noticeable.

Determine the score based on fuzzy comprehensive evaluation principle. For the whole evaluated pictures and single symbols, the fuzzy comprehensive evaluation result is fuzzy set $B$ with the unit factors judgment matrix $R$ and weights $A$ shown in Eq.7.

$$
B=A \times R=\left(a_{1}, a_{2}, a_{3}, a_{4}, a_{5}\right) \times\left[\begin{array}{llll}
v_{11} & v_{12} & v_{13} & v_{14} \\
v_{21} & v_{22} & v_{23} & v_{24} \\
v_{31} & v_{32} & v_{33} & v_{34} \\
v_{41} & v_{42} & v_{43} & v_{44} \\
v_{51} & v_{52} & v_{53} & v_{54}
\end{array}\right]
$$

According to the principle of maximum membership, the maximum of Matrix $B$ is the fuzzy comprehensive evaluation value.

Evaluation results. The fuzzy evaluation results of whole charts and single symbol are shown from Fig 6 to Fig 10.

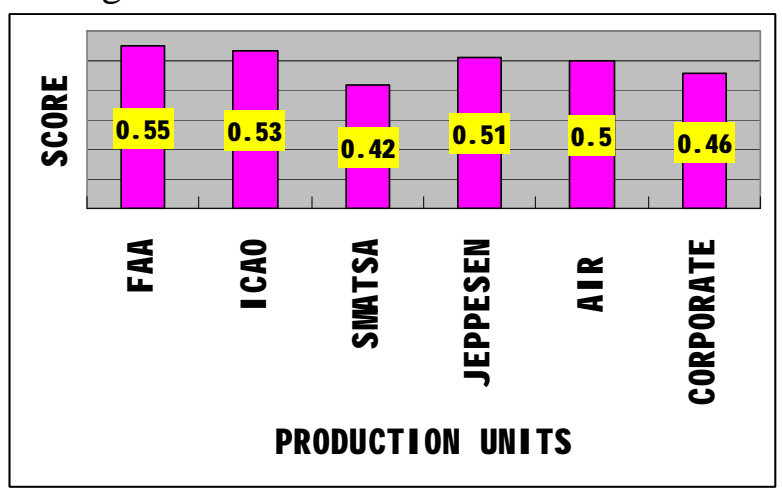

Fig.6 Assessment of whole chart

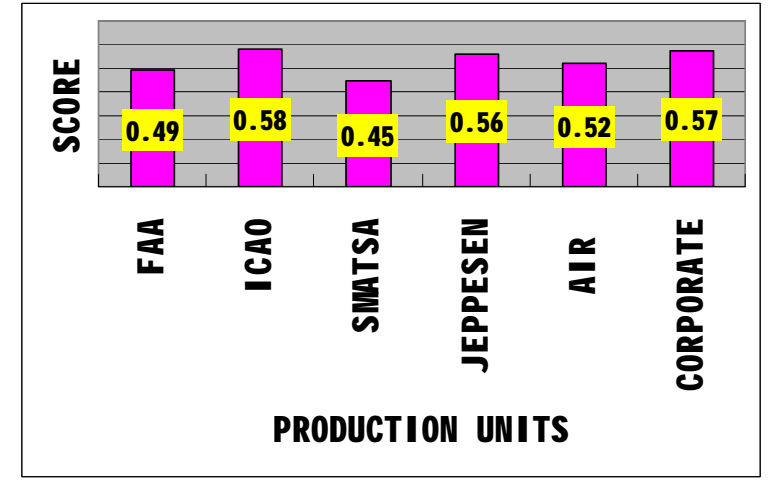

Fig.7 Assessment of airport symbol

From the aggregated data and results, as shown in Fig 6,FAA gets the higher evaluation value ,because the VFR chart of FAA obtains higher score in two index(information transmission and symbol recognition) of higher weight, which is consistent with the actual, if an aeronautical could well provide information and symbol recognition, it will get evaluation. The symbol shape of CORPORATE is the same as FAA, but its color configuration is bad and the whole display effect is poor ; The symbol design of ICAO is very image, but the kinds of color is too many, which gives users a low intimacy; JEEPESEN has too much textual representation, not make full use of symbols to convey information.

In the assessment of airport symbol, as shown in Fig 7, ICAO, JEPPESEN and CORPORATE have the higher evaluation value. Watch the symbols, we can find the airport symbol of the three units mainly highlight the runway, and the rest all put a small rectangular in a circular, and they are easy to be mistaken as NDB symbols or VOR symbols so the score is low. 


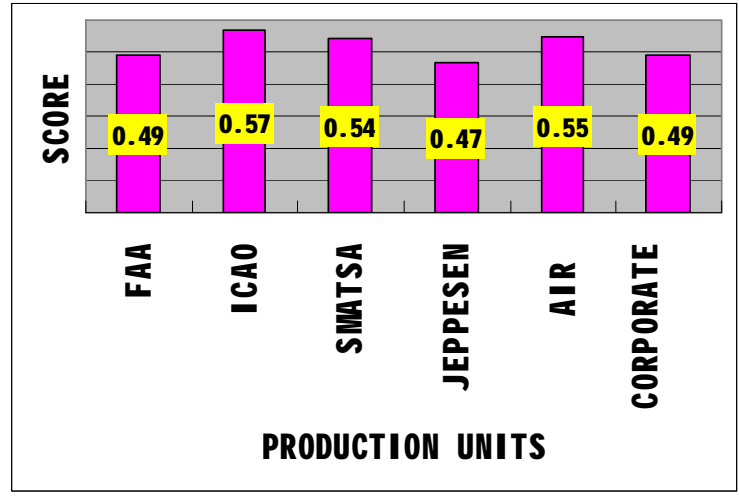

Fig.8 Assessment of obstacle symbol

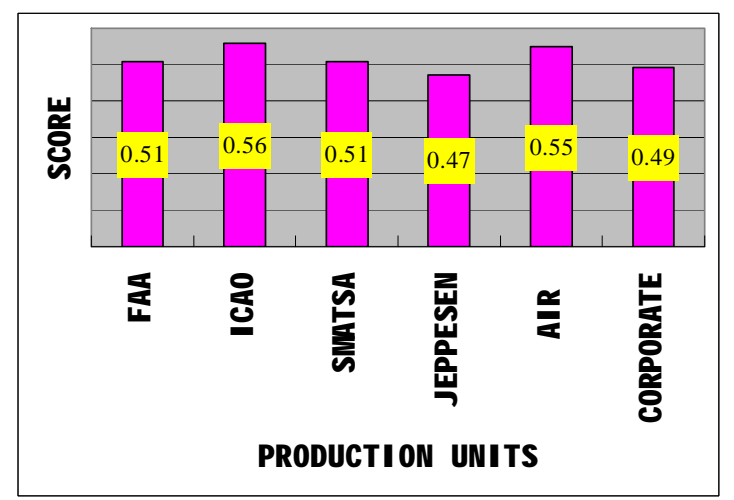

Fig.9 Assessment of NDB symbol

In the assessment of obstacle symbol, as shown in Fig 8, ICAO gets the highest score, because its design of obstacle symbol is very image. The rest symbol shape is similar, but the color of SMATSA and AIR is red, with the warning role, they are easy to recognize and have the higher score.

In the assessment of NDB symbol, as shown in Fig 9, ICAO and AIR have the higher score. Watch the symbols, we can find the NDB symbol shape of the six units are essentially the same, the difference lies mainly in that the integrity of the NDB symbols of ICAO and AIR are not destroyed, and colors are in turn yellow and blue, easy to be identified.

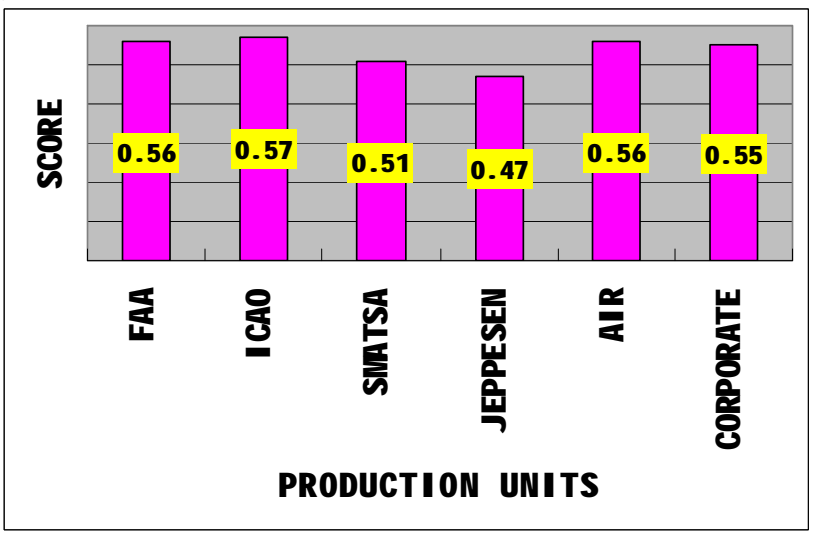

Fig.10 Assessment of VORDME symbol

In the Assessment of VORDME symbol, as shown in Fig 10, only the JEEPESEN gets the lower score, because its symbols do not apply the conventional shape to design, the symbol shape of other units essentially the same, just different shades, so the score is similar.

We can get some principles of designing symbol from the above analysis:

1) The color contrast of different type symbol should not too large. The symbol color contrast which has no similar elements should not be too big but be coordinate. For similar elements distinguish them from brightness and saturation, but they are quietly elegant in general.

2) The design should be simple and textual representation should not be too much, try to use symbols to express, but chart also should use a number of ancillary information (such as name or frequency) to help symbol recognition.

3) The shape of symbol should as far as possible be similar with the chart element that represents, the symbol is easy to be identified.

4) The symbols displayed on the chart should maintain the integrity. When other lines have to pass through the symbol, they should be in relatively shallow color lining the symbol below.

\section{Conclusion}

This article organize the pilots to attend the visual perception test of VFR chart, then processes data based on fuzzy evaluation and gets the evaluation result. Further, several principles of VFR charts and symbols in designing are get in contrast to the VFR chart quality. It provides a theoretical basis for the establishment and evaluation of VFR chart symbol library. 


\section{Acknowledgment}

This work is supported by National Nature Science Foundation under Grant No. 61039001 and 60832011. And it is also supported by National Technology Support Program under Grant No. 2011BH24B10.

\section{References}

[1] Yeh, M. and Chandra, D.C. Designing and Evaluating Symbols for Electronic Displays of Navigation Information: Symbol Stereotypes and Symbol-Feature Rules (DOT/FAA/AR-05/48; DOT-VNTSC-FAA-05-16).Washingtn, DC. U.S. Department of Transportation, Federal Aviation Administration, 2005.

[2] Yeh, M. and Chandra, D.C. Pilot Identification of Symbols and an Exploration of Symbol Design Issues for Electronic Displays of Aeronautical Charting Information. (DOT/FAA/AR-07/37;DOT-VNTSC-FAA-07-07).Washington, DC. U.S. Department of Transportation, Federal Aviation Administration, 2007.

[3] Aimin Meng: Civil Aviation of China,Vol. 51(2005)No.03., p.59.

[4] Xinming Zheng: Air Traffic Management,Vol. 37(2010)No.01, p.16.

[5] Xinming Zheng: Air Traffic Management, Vol. 28 (2008)No.10, p.37.

[6] Yufen Chen : Journal of the PLA institute of surveying and mapping, Vol. 36 (1995)No.02, p.145.

[7] Chao Xie: Research on the Eleetronic ToPograPhic MaP Symbol System(Master, PLA information engineering university, China 2006). 